\title{
Behavioural addictions matter
}

\author{
More research, and dedicated funding, is needed to understand and \\ successfully treat compulsive habits, says Marc Potenza.
}

W hat behaviours can be considered addictions? Gambling, gaming, Internet use, sex, shopping and eating can become excessive, but whether they should be labelled as addictions is an ongoing debate.

In the most recent, fifth edition of the Diagnostic and Statistical Manual of Mental Disorders (DSM-5) - a book published by the American Psychiatric Association in 2013 that defines and classifies mental health conditions - gambling disorder was moved from its category of 'Impulse-control disorders not elsewhere classified' to 'Substance-related and addictive disorders'. This represents a significant shift from a view that has prevailed since the 1980s that addictions are disorders involving compulsive drug use, and multiple non-substance-related behaviours may now be considered addictions ${ }^{1}$.

Gambling disorder is currently the only nonsubstance condition that is listed as an addiction in the DSM-5, although a work group proposed that Internet gaming disorder (IGD) warrants additional research. Multiple aspects of IGD remain controversial, including to what extent the Internet may be the vehicle versus the focus of a disorder, and, if a broader 'Internet-use disorder' is to be accepted, the extent to which use represents an addiction. The work group focused on gaming because it was the most well studied and arguably problematic form of Internet use at the time ${ }^{2}$, but behaviours such as social networking and pornography viewing are also under scrutiny. Such uses of the Internet also appear clinically relevant: problematic online social networking, for example, has been linked to poor emotional regulation and problems with alcohol use among university students ${ }^{3}$. Given that more people are growing up with digital technology, considering a broader range of Internet-related activities as potentially addictive seems important for addiction researchers.

\section{DEFINING BIZARRE BEHAVIOUR}

But even if such diagnoses were to be accepted, the question of where to draw the line between abnormal and normal behaviours is still up for debate and has contributed to wide variations in prevalence estimates for problematic Internet use ${ }^{2}$. Currently, the DSM-5 uses a more stringent threshold for diagnosing gambling disorder (it must meet 4 or more inclusionary criteria out of 9) or Internet gaming disorder ( 5 or more out of 10) than it does for diagnosing substance-use disorders ( 2 or more inclusionary criteria out of 11); we must take care not to underestimate how widespread such non-substance behaviours are and the negative impact that they can have on public health.

Another controversial topic is sex addiction. Formal criteria for hypersexual disorder have been proposed and tested ${ }^{4}$, but the condition was not included in the DSM-5. As with the other behavioural addictions, debate exists about where to set the threshold between normal and abnormal levels of sexual activity. Nevertheless, similarities in cognitive and biological changes involving craving and reward circuitry have been noted between compulsive sexual behaviours and substance and gambling addictions, and scales assessing addiction-like features such as craving seem relevant to aspects of sexual behaviours. A better understanding of aetiological and associated factors, such as to what extent psychological and biological determinants linked to gambling and substance addictions also relate to hypersexuality, should help classification efforts and promote the development of targeted treatments.

Other behaviours, including excessive eating and shopping, are also sometimes considered addictions. Of note, patients receiving dopamine-boosting treatment for Parkinson's disease have sometimes developed excessive eating, shopping, sex and gambling habits, suggesting that there may be a biological link that drives all of these behaviours. But there are nuances: although obesity has been found to share biological features with substance addictions, the variety of ways that the condition manifests itself suggests that only a subset of individuals with obesity may be characterized by food addiction. In particular, individuals with bingeeating disorder are likely to meet food-addiction criteria, suggesting similarities with gambling disorder and substance-use disorders. If foods are demonstrated to have addictive potential, it would be important to identify the specific foods or food components and implement relevant public-health policies and interventions.

While experts debate which non-substance disorders may constitute addictions, people continue to exhibit problematic behaviours. Thus, more research is needed to better understand the epidemiological, clinical, neurobiologi$\mathrm{cal}$, genetic and cultural features to help prevent and treat behavioural addictions. Research was paramount in compiling the DSM-5, and a similar process should be used in writing the 11th edition (due in 2017) of the World Health Organization's International Classification of Diseases. But for this to happen, funding agencies must prioritize research into non-substance addictions. In the United States, the National Institutes of Health includes departments focusing on drugs and alcohol, but none that target behavioural addictions. The creation of a national institute on behavioural addictions would help to advance research in this area. In France, the government requires addiction treatment centres to provide care for people with behavioural addictions. Thus, how we classify these behaviours has direct clinical implications, and there is an important need to understand how best to prevent behavioural addictions and help people who experience harm related to them.

Marc Potenza is director of the Center of Excellence in Gambling Research at Yale University in New Haven, Connecticut. e-mail:marc.potenza@yale.edu

1. Potenza, M. N. Addiction 101, 142-151 (2006).

2. Petry, N. M. \& O'Brien, C. P. Addiction 108, 1186-1187 (2013).

3. Hormes, J. M., Kearns, B. \& Timko, C. A. Addiction 109, 2079-2088 (2014).

4. Reid, R. C. et al. J. Sex Med. 9, 2868-2877 (2012). 